\title{
REDUÇÃO DA CARGA ORGÂNICA DE DEJETOS LÍQUIDOS DE SUÍNOS POR MACRÓFITAS AQUÁTICAS
}

\author{
Camila Dias Pinaffi, Carlos Henrique dos Santos \\ Universidade do Oeste Paulista - UNOESTE, Presidente prudente, SP. E-mail: camila pinaffi@hotmail.com
}

\begin{abstract}
RESUMO
O alto teor de carga orgânica dos dejetos suínos acarreta em contaminação das águas. As plantas aquáticas representam uma possibilidade de manejo destes dejetos. Neste contexto, o estudo objetivou avaliar o potencial das macrófitas aguapé, alface d'água e salvínia na redução da carga orgânica dos dejetos. O experimento ocorreu em ambiente aberto no Campus II/UNOESTE, estruturado em delineamento em blocos casualizados, com 4 tratamentos e 4 repetições, totalizando 16 parcelas. Foi utilizado dejeto de líquido de suíno (DLS) diluído em $50 \%$ de água, e distribuído em caixas de PVC. Os tratamentos adotados foram: T1 = DLS; T2 = DLS+aguapé; T3 = DLS+alface d'água; T4 = DLS+salvínia. Os parâmetros avaliados foram: demanda química e bioquímica de oxigênio (DQO e DBO). Foi determinada a eficiência e aplicados modelo de regressão. Os resultados obtidos indicam que o aguapé proporcionou maior redução de DQO e DBO, destacando-o como mais eficiente na limpeza dos dejetos.
\end{abstract}

Palavras-chave: matéria orgânica, suinocultura, fitorremediação.

\section{REDUCTION OF THE ORGANIC LOAD OF SWINE MANURE BY AQUATIC MACROPHYTES}

\begin{abstract}
The high organic load content of swine manure results in water contamination. The aquatic plants represent a possibility of handling these wastes. In this context, the objective of this study was to evaluate the potential of macrophytes, water lettuce and salvinia in the reduction of the organic load of the wastes. The experiment was carried out in an open environment in Campus II/UNOESTE, structured in a randomized block design, with 4 treatments and 4 replications, totaling 16 plots. Swine liquid waste (DLS) diluted in 50\% water was used and distributed in PVC boxes. The treatments adopted were: $\mathrm{T} 1=\mathrm{DLS} ; \mathrm{T} 2=\mathrm{DLS}+$ water; $\mathrm{T} 3=\mathrm{DLS}+$ water lettuce; $\mathrm{T} 4=$ DLS + salvinia. The parameters evaluated were: chemical and biochemical oxygen demand (COD and BOD). We determined the efficiency and applied regression model. The results indicate that the water source provided a greater reduction of COD and BOD, highlighting it as more efficient in cleaning the waste.
\end{abstract}

Keywords: organic matter, swine breeding, phytoremediation.

\section{INTRODUÇÃO}

O desenvolvimento da suinocultura elevou produção de dejetos, que pela ausência de tratamento adequado, se transformou em grande fonte poluidora, principalmente, dos mananciais de água. Um suíno de 60 quilos, por exemplo, é capaz de produzir uma carga orgânica de $0,136 \mathrm{~kg}_{\text {.dia }}{ }^{-1}$ de demanda bioquímica de oxigênio (DBO), que é um dos principais indicadores do potencial poluidor ao meio ambiente. Isto representa quatro vezes ao equivalente populacional humano (CAMPAGANRO et al., 2007). 
O manejo inadequado dos resíduos da suinocultura (extravasamento de esterqueiras, aplicação excessiva no solo) pode ocasionar a contaminação de rios (como a eutrofização), de lençóis subterrâneos (como aumento da concentração do íon nitrato), do solo (patógenos e excesso de nutrientes, dentre outros) e do ar (como emissões gasosas) (KUNZ et al., 2005).

Diante dessas evidências, torna-se importante a proposição de um sistema de tratamento de efluentes oriundos de granjas de suinocultura, a fim de minimizar os efeitos prejudiciais aos ecossistemas aquáticos e a carga orgânica desse material.

Estudos demonstram a eficiência das plantas aquáticas no tratamento de efluentes e os teores de absorção de poluentes (OLIVEIRA et al. 2000; BALLEM et al. 2007; GONÇALVES JÚNIOR et al., 2008). A alta capacidade de produção de biomassa de macrófitas aquáticas em ambientes com altos níveis de nutrientes e carga orgânica tem gerado interesse na comunidade científica em sua utilização em sistemas de tratamento (PISTORI, 2009).

Estas plantas têm a capacidade de realizarem filtração, degradação e assimilação de poluentes minerais e orgânicos e retenção de nutrientes (CAMARGO et al., 2003). Neste sentido, o presente estudo tem como objetivo avaliar o potencial das plantas aquáticas aguapé (Eichhornia crassipes), alface d'água (Pistia stratiotes) e salvínia (Salvinia auriculata) na redução da carga orgânica (demandas química e bioquímica de oxigênio - DQO e DBO) de dejetos líquidos de suínos.

\section{MATERIAL E MÉTODOS}

O experimento foi instalado em uma área de ambiente aberto no Campus II da Universidade do Oeste Paulista (UNOESTE), Presidente Prudente/SP, durante o período de março a setembro de 2016. A área está localizada a $475 \mathrm{~m}$ de altitude em uma região de clima definido como Aw, conforme classificação de Köppen. As coordenadas geográficas do local são: Latitude $22^{\circ} 07^{\prime} \mathrm{S}$ e Longitude $51^{\circ} 09^{\prime} \mathrm{O}$.

Para compor os tratamentos utilizou-se o dejeto líquido de suínos (DLS) da suinocultura do centro zootécnico, do Campus II/UNOESTE, no qual foi introduzido em sistemas de tratamentos constituídos de 16 recipientes de policloreto de vinila (PVC) com volume de $0,32 \mathrm{~m}^{3}$, sendo utilizado apenas $0,25 \mathrm{~m}^{3}$ por recipiente, a fim de assegurar uma margem caso a ocorrência de chuvas.

Os tratamentos adotados no estudo foram: T1 = Testemunha - 50\% de DLS e 50\% de água; $\mathrm{T} 2=50 \%$ de DLS e $50 \%$ de água $+E$. crassipes $; \mathrm{T} 3=50 \%$ de DLS e $50 \%$ de água $+P$. stratiotes $; \mathrm{T} 4=$ $50 \%$ de DLS e $50 \%$ de água + S. auriculata, distribuídos em delineamento em blocos casualizados, em parcelas subdivididas, com quatro repetições. As parcelas constituem os tratamentos (sem e com a presença de plantas aquáticas) e as subparcelas, os períodos de coleta do efluente para análise química (0 dia - entrada; 30 dias - saída).

O DLS utilizado foi submetido previamente à caracterização química, realizada em triplicata, e conforme método proposto por Eaton et al. (2005). As concentrações da demanda química de oxigênio e bioquímica de oxigênio ( $\mathrm{DQO}$ e $\mathrm{DBO}_{5} 20^{\circ} \mathrm{C}$ ), em mg L ${ }^{-1}$, obtidos foram, respectivamente, $1428,29 \pm 188,78$ e $842,04 \pm 28,43$.

As macrófitas aquáticas foram apanhadas em lagos lênticos de propriedades localizadas na região do Oeste Paulista/SP. Previamente, as plantas foram submetidas à adaptação ambiental em caixas com água, durante uma semana. Em seguida, iniciou-se o tratamento, com a introdução das plantas nas unidades experimentais, com uma ocupação equivalente a $80 \%$, seguindo relatos de Henry-Silva e Camargo (2008). As coletas mensais de efluente foram realizadas em três ciclos subsequentes de manejo, totalizando três ciclos de avaliação (Março/Abril, Maio/Junho, 
Agosto/Setembro de 2016). Após a realização de cada ciclo de 30 dias, o efluente foi substituído, bem como, todas as plantas aquáticas.

Durante o período experimental, foram coletadas semanalmente 16 amostras do efluente, sendo uma amostra por caixa, acondicionada em garrafas plásticas descartáveis com tampa lacre de $500 \mathrm{ml}$. Procedeu-se a composição destas amostras em função das restrições do equipamento de análise, ou seja, as amostras coletadas de cada repetição foram homogeneizadas a fim de obter uma amostra composta de cada tratamento.

Os parâmetros avaliados foram DQO e DBO, conforme Eaton et al. (2005). A determinação da DQO foi obtida pelo método que corresponde a oxidação química da matéria orgânica, obtida pelo uso de um agente oxidante forte, o dicromato de potássio $\left(\mathrm{K}_{2} \mathrm{Cr}_{2} \mathrm{O}_{7}\right)$ em meio ácido, com temperatura elevada pelo bloco digestor, marca Alfakit. Durante a determinação da DQO, a matéria orgânica é convertida a $\mathrm{CO}_{2}$ e $\mathrm{H}_{2} \mathrm{O}$. A procedência da leitura, em absorbância, foi realizada através do espectrofotômetro marca CELM. Para obtenção da $\mathrm{DBO}_{5} 20^{\circ} \mathrm{C}$ foi utilizado o método respirométrico simplificado, OxiTop ${ }^{\oplus}$. As determinações foram realizadas no primeiro dia (entrada) e ao final dos 30 dias (saída). As análises foram efetuadas no laboratório de análises de água, no Bloco Q, Campus II/UNOESTE.

A eficiência dos tratamentos na redução dos parâmetros foi calculada conforme a equação:

$$
E(\%)=\frac{C i-C f}{C i} \times 100
$$

Em que: $\mathrm{E}=$ Eficiência de remoção (\%); $C i=$ Concentração inicial $\left(\mathrm{mg} \mathrm{L}^{-1}\right) ; C f=$ Concentração final $\left(\mathrm{mg} \mathrm{L}^{-1}\right)$. Considerou-se os valores de concentração inicial, o valor obtido nas análises da entrada, e os de concentração final, o valor obtido nas análises da saída.

Os resultados também foram submetidos à análise de regressão.

\section{RESULTADOS E DISCUSSÃO}

De acordo com Von Sperling (2005), a DQO e a DBO retratam, de uma forma indireta, o teor de matéria orgânica no corpo hídrico, sendo, portanto, uma indicação do potencial do consumo do oxigênio dissolvido no sistema; representam os parâmetros de maior importância na caracterização do grau de poluição de um corpo d'água.

A partir dos valores de DQO obtidos no primo ciclo (Tabela 1), constatou-se que, nas duas coletas realizadas houve redução expressiva deste parâmetro do início do tratamento para o final. Apesar da pequena diferença na redução observada entre os tratamentos, observa-se que os manejos com plantas apresentaram expressivos percentuais de remoção da DQO, sendo de $84,3 \%$ para $E$. crassipes, $85,4 \%$ para a $P$. stratiotes e $87,2 \%$ para a $S$. auriculata, revelando que a presença das plantas aquáticas auxiliou na degradação da matéria orgânica presente no efluente. Shah et al. (2015) relataram que grande parte da redução da DQO nas águas residuais pode ser atribuída aos microrganismos, possivelmente estabelecendo uma relação simbiótica com as plantas.

No segundo ciclo de avaliação (Tabela 1) houve redução na concentração de DQO dos efluentes manejados com as plantas aquáticas, com destaque ao tratamento com $E$. crassipes, que alcançou $80,1 \%$ de remoção. Reidel et al. (2005), ao avaliar o desenvolvimento da tilápia do Nilo em efluente de frigorífico, tratado em sistema com E. crassipes, registrou valores médios de DQO no efluente da lagoa de polimento da indústria de $174,51 \mathrm{mg} \mathrm{L}^{-1}$ e redução de DQO de 73,88 , 72,76 e $87,4 \%$ no sistema com E. crassipes, para os tempos de detenção hidráulico de 5,7 e 10 dias, respectivamente. Estudo realizado por Ballem et al. (2007), no qual foi avaliado a eficiência 
do sistema lagoa de E. crassipes na remoção complementar de DQO de dejetos líquidos de suínos pré-tratados em reator aeróbico de biogrânulos, constatou-se eficiência de 79,2\% para DQO total.

No terceiro ciclo (Tabela 1), semelhantemente ao anterior, também houve redução nos valores de DQO, com destaque ao tratamento com E. crassipes, com taxa de remoção de $75,4 \%$.

A E. crassipes obteve destaque em todos os ciclos, seguida da P. stratiotes. Os trabalhos que utilizaram a $P$. stratiotes para o tratamento de esgoto doméstico utilizou DQO inicial na faixa de 270 a 550 mg/L (ZIMMELS; KIRZHNER; MALKOVSKAJA, 2006) e para o tratamento de efluente de piscicultura utilizou DQO inicial de $206 \mathrm{mg} / \mathrm{L}$ (AKINBILE; YUSOFF, 2012). Nos dois casos os resultados foram satisfatórios na redução da carga orgânica dos efluentes.

TABELA 1 - Valores de DQO dos ciclos de tratamentos com dejetos líquidos de suínos, na presença de E. crassipes, P. stratiotes, S. auriculata. Presidente Prudente, 2016.

\begin{tabular}{ccccccccccc}
\hline \multicolumn{10}{c}{ DQO (mg L } \\
\hline \multirow{2}{*}{ Tratamentos } \\
& Entrada & Saída & E (\%) & Entrada & Saída & E (\%) & Entrada & Saída & E (\%) \\
\hline Testemunha & 810,33 & 133,67 & 83,5 & 747,00 & 210,33 & 71,8 & 960,67 & 407,00 & 57,6 \\
E. crassipes & 787,00 & 123,67 & 84,3 & 720,33 & 143,67 & 80,1 & 853,67 & 210,33 & 75,4 \\
P. stratiotes & 733,67 & 107,00 & 85,4 & 700,33 & 263,67 & 62,4 & 957,00 & 527,00 & 44,9 \\
S. auriculata & 887,00 & 113,67 & 87,2 & 687,00 & 243,67 & 64,5 & 960,67 & 940,33 & 2,1 \\
\hline
\end{tabular}

E (\%): Eficiência de remoção

A análise de regressão das médias, do período inicial (entrada) e do final (saída), compila os três ciclos de avaliação e permitem uma observação mais ampla dos resultados (Figura 1). Houve uma expressiva redução da média obtida na entrada para a média obtida na saída. De acordo com a curva de tendência formada com as médias da entrada constata-se que os valores de DQO aumentaram conforme o ciclo de avaliação, sendo que no terceiro ciclo, realizado no mês de agosto, a concentração de DQO foi superior.

Em relação à curva da média da saída nota-se um incremento da DQO nos ciclos avaliados, o que sugere que no terceiro ciclo, caracterizado por temperaturas mais amenas, a minimização da DQO foi menos expressiva. Logo, provavelmente, a condição climática representa um fator determinante na remoção de matéria orgânica pelas macrófitas aquáticas. Além disso, de acordo com Shah et al. (2015), o crescimento das macrófitas proporciona um elevado desempenho para remoção de DQO principalmente por causa do sistema radicular bem desenvolvido diante da adsorção dos particulados nas raízes. No presente estudo, no primeiro ciclo de avaliação a elevada produção de biomassa pode ter proporcionado grande redução da DQO. Tonetto (2015), ao estudar a capacidade de um sistema wetland para o polimento de efluente de indústria alimentícia e avaliar a capacidade de biodegradabilidade de acordo com as condições climáticas, constatou que a eficiência da remoção da DQO apresentou maior porcentagem no verão $(51,48 \%)$. 
FIGURA 1 - Valores médios de DQO, dos dois períodos de avaliação, obtidos nos 1, 2ㅇ e 3을 ciclos

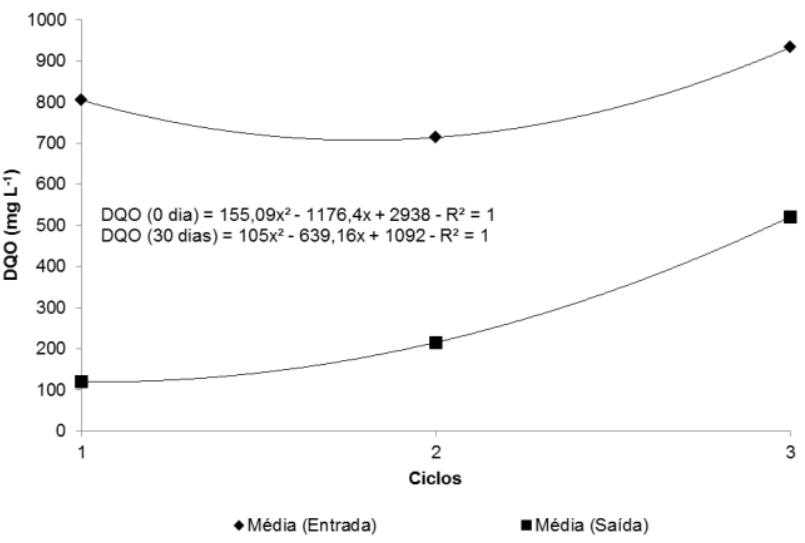

Fonte: Os autores (2017)

Conforme os valores de DBO obtidos no primeiro ciclo (Tabela 2), observa-se também pequena diferença entre os tratamentos, sendo aquele manejado com $E$. crassipes houve a maior eficiência na remoção da DBO (92,6\%), indicando um melhor desempenho desta espécie para remoção de matéria orgânica neste tratamento. Shah et al. (2015), ao avaliar o desempenho de três macrófitas (aguapé, lentilha e alface d'água) no tratamento de águas residuais do município, obteve a redução média do valor de DBO do efluente de 50,6\% para a E. crassipes e 33,4\% para às demais espécies. Estes autores ainda observaram que a remoção da DBO ocorre principalmente nos primeiros 10 dias, e isso pode ser atribuído a uma absorção superior da planta neste período.

Os valores de DBO, obtidos no segundo ciclo (Tabela 2), mostram que o potencial de remoção pela espécie $E$. crassipes foi mais expressivo $(76,4 \%)$. Além disso, constata-se que o tratamento testemunha também diminuiu a concentração de DBO, porém com 55,3\%. Deste modo, apenas a eficiência obtida com E. crassipes está de acordo com os parâmetros da Resolução CONAMA 430/2011, que define como limite mínimo de remoção de 60\% de DBO.

No terceiro ciclo (Tabela 2), observa-se também expressiva redução nas concentrações de DBO em todos os tratamentos, com destaque para a espécie $E$. crassipes com remoção de $88,6 \%$.

TABELA 2 - Valores de DBO dos ciclos de tratamentos com dejetos líquidos de suínos, na presença de E. crassipes, P. stratiotes, S. auriculata. Presidente Prudente, 2016.

\begin{tabular}{|c|c|c|c|c|c|c|c|c|c|}
\hline \multicolumn{10}{|c|}{$\mathrm{DBO}\left(\mathrm{mg} \mathrm{L}^{-1}\right)$} \\
\hline \multirow{2}{*}{ Tratamentos } & \multicolumn{3}{|c|}{ 10 CICLO } & \multicolumn{3}{|c|}{ 2o CICLO } & \multicolumn{3}{|c|}{ 3으 CICLO } \\
\hline & Entrada & Saída & $E(\%)$ & Entrada & Saída & $E(\%)$ & Entrada & Saída & $E(\%)$ \\
\hline Testemunha & 480,24 & 73,96 & 84,6 & 397,91 & 177,94 & 55,3 & 520,44 & 87,91 & 83,1 \\
\hline E. crassipes & 377,91 & 27,94 & 92,6 & 287,91 & 67,94 & 76,4 & 419,94 & 47,94 & 88,6 \\
\hline P. stratiotes & 427,91 & 74,90 & 82,5 & 237,91 & 177,94 & 25,2 & 500,34 & 147,91 & 70,4 \\
\hline S. auriculata & 560,64 & 65,96 & 88,2 & 217,91 & 142,94 & 34,4 & 520,44 & 279,24 & 46,4 \\
\hline
\end{tabular}

E (\%): Eficiência de remoção

Através da curva de tendência formada com as médias da entrada (Figura 2) observa-se que o primeiro e o terceiro ciclos apresentaram maiores valores de DBO, ou seja, maior teor de matéria orgânica no efluente. De acordo com os dados da curva da média de saída nota-se uma elevação nos valores de DBO dos segundo e terceiro ciclos quando comparados ao primeiro, o que Colloquium Agrariae, vol. 13, n. Especial, Jul-Dez, 2017, p. 181-188. ISSN: 1809-8215. DOI: 10.5747/ca.2017.v13.nesp.000191 
revela, novamente, que o terceiro ciclo, caracterizado por temperatura mais elevada, a redução da DBO foi mais expressiva.

FIGURA 2 - Valores médios de DBO, dos dois períodos de avaliação, obtidos nos 1ำ, 2으 e 3ำ ciclos

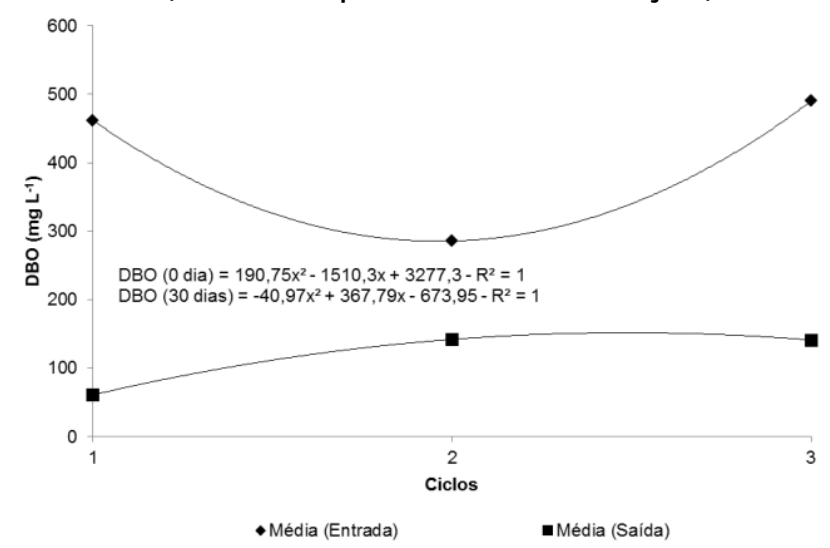

Fonte: Os autores (2017)

\section{CONCLUSÃO}

Conclui-se que houve redução expressiva da DQO e DBO no efluente proporcionada principalmente pela $E$. crassipes, dessa forma, as macrófitas aquáticas estudadas, com destaque para a E. crassipes, podem ser aliadas na limpeza de dejetos de suínos, dando aos produtores uma alternativa de manejo para adequação ambiental de seus sistemas de criação de suínos.

\section{REFERÊNCIAS}

AKINBILE, C. O.; YUSOFF, M. S. Assessing water hyacinth (Eichhornia crassipes) and lettuce (Pistia stratiotes) effectiveness in aquaculture wastewater treatment. International Journal of Phytoremediation, v. 14, n. 3, p. 201-211, 2012. https://doi.org/10.1080/15226514.2011.587482

BALLEM, A.; AITA, C; GIACOMINI, S. J.; HÜBNER, A. P.; PUJOL, S.; LEAL, A. J. Eficiência do sistema lagoa de aguapés na remoção complementar de $\mathrm{DQO}$ e $\mathrm{N}$ de dejetos líquidos de suínos prétratados em reator aeróbico de biogrânulos. In: CONGRESSO BRASILEIRO DE CIÊNCIA DO SOLO, 21., 2007, Gramado. Anais... Gramado: [s.n.], 2007.

CAMARGO, A. F. M.; HENRY-SILVA, G. G.; PEZZATO, M. M. Crescimento e produção primária de macrófitas aquáticas em zonas litorâneas. In: HENRY, R. (Ed.) Ecótonos nas interfaces dos ecossistemas aquáticos. São Carlos: Fundibio/Rima, p. 213-232, 2003.

CAMPAGANRO, V. F; DAL BOSCO, T. C; EVARINE, J. A.; IOST, C.; DALLAGO, R. C.; GOMES, S. D. Caracterização físico-química de dejetos suínos em sistemas de criação diferenciados e potenciais riscos ambientais relacionados. In: XVI Semana de Biologia da UNIOESTE, 2007.

CONAMA - CONSELHO NACIONAL DO MEIO AMBIENTE. Resolução Conama no 430 de 13 de maio de 2011. Dispõe sobre as condições e padrões de lançamento de efluentes, complementa e altera a Resolução n. 357, de 17 de março de 2005, do Conselho Nacional do Meio Ambiente-CONAMA. Diário Oficial da União, Brasília, DF, n. 92, de 16/05/2011, p.89. 
EATON, A. D.; CLESCERI, L. S.; RICE, E. W.; GREENBERG, A. E.; FRANSON, M. A. H. (eds.). Standard methods for the examination of water and wastewater: Centennial Edition. 21. Ed. American Public Health Association. 1600 p. 2005.

GONÇALVES JÚNIOR, A. C.; LINDINO, C. A.; ROSA, M. F.; R BARICCATTI, R.; GOMES, G. D. Remoção de metais pesados tóxicos cádmio, chumbo e cromo em biofertilizante suíno utilizando macrófita aquática (Eichornia crassipes) como bioindicador. Acta Scientiarum. Technology, v. 30, n. 1, p. 914, 2008. https://doi.org/10.4025/actascitechnol.v30i1.3179

HENRY-SILVA, G. G.; CAMARGO, A. F. M. Tratamento de efluentes de carcinicultura por macrófitas aquáticas flutuantes. Revista Brasileira de Zootecnia, v. 37, n. 2, p. 181-188, 2008. https://doi.org/10.1590/S1516-35982008000200002

KUNZ, A.; HIGARASHI, M. M.; OLIVEIRA, P. A. Tecnologias de manejo e tratamento de dejetos de suínos estudadas no Brasil. Cadernos de Ciência \& Tecnologia, Brasília, v. 22, n. 3, p. 651-665, set./dez. 2005.

OLIVEIRA, R. A.; DENÍCULI, W.; ITABORAHY, C. R.; CECON, P. R. Redução da demanda bioquímica de oxigênio de águas residuárias da suinocultura com emprego da macrófita aquática. Revista Brasileira de Engenharia Agrícola e Ambiental, v. 4, n. 1, p. 81-86, 2000. https://doi.org/10.1590/S1415-43662000000100015

PISTORI, R. E. T. Crescimento das macrófitas aquáticas flutuantes Eichhornia crassipes(Mart.) Solms, Pistia stratiotes L. e Salvinia molesta(Mitchell) em diferentes concentrações de nutrientes. 2009. 63 f. Tese (Doutorado em Aquicultura) - Universidade Estadual Paulista, Jaboticabal, São Paulo.

ReIDEL, A.; DAMASCENO, S.; ZenATTI, D. C.; SAMPAIO, S. C.; FEIDEN, A.; QUeIROZ, M. M. F. Utilização de efluente de frigorífico, tratado com macrófita aquática, no cultivo de tilápia do Nilo. Revista Brasileira Engenharia Agrícola e Ambiental, Campina Grande, v. 9, (supl), p. 181-185, 2005.

SHAH, M.; HASHMI, H. N.; GHUMMAN, A. R.; ZEESHAN, M. Performance assessment of aquatic macrophytes for treatment of municipal wastewater. Journal of the South African Institution of Civil Engineering, v. 57, n. 3, p. 18-25, 2015. https://doi.org/10.17159/2309-8775/2015/V57N3A3

TONETTO, J. F. Avaliação sazonal do potencial de biodegradabilidade e remoção de cargas em efluente industrial via wetland construído. 2015. 62f. Dissertação (Mestrado) - Centro Universitário UNIVATES, Lajeado, 2015.

VON SPERLING, M. Introdução à qualidade das águas e ao tratamento de esgotos. 3. ed. Belo Horizonte: Departamento de Engenharia Sanitária e Ambiental. Universidade Federal de Minas Gerais, 2005. 
ZIMMELS, Y.; KIRZHNER, F.; MALKOVSKAJA, A. Application of Eichhornia crassipes and Pistia stratiotes for treatment of urban sewage in Israel. Journal of Environmental Management, v. 81, n. 4, p. 420-428, 2006. https://doi.org/10.1016/j.jenvman.2005.11.014 